\title{
Bartter Syndromes and Other Salt-Losing Tubulopathies
}

\author{
Robert Kleta $^{\mathrm{a}, \mathrm{b}}$ Detlef Bockenhauer ${ }^{\mathrm{c}}$ \\ a Section on Human Biochemical Genetics, Medical Genetics Branch, National Human Genome Research \\ Institute, and ${ }^{\mathrm{b}}$ Office of Rare Diseases, Office of the Director, National Institutes of Health, Bethesda, Md., USA; \\ ${ }^{\mathrm{c}}$ Great Ormond Street Hospital for Children NHS Trust, Great Ormond Street, London, UK
}

\section{Key Words}

Renal Fanconi syndrome · Bartter syndrome · Gitelman syndrome $\cdot$ Pseudohypoaldosteronism $\cdot$ Sodium transport

\begin{abstract}
Genetic studies into rare inborn errors of renal tubular sodium handling in man have brought many interesting, sometimes surprising insights into how we can maintain our bodies' electrolytes and fluids homeostasis. The cloning and identification of sodium transporting genes and proteins like NHE3, NKCC2, ROMK, CLCNKB, NCC, and $\mathrm{EnaC}$ has considerably improved our understanding of renal salt handling. Subsequently, studies of genetically engineered animals provided even more insight into the complex renal physiology. The recent discovery of the WNK kinases as regulators and integrators of specific renal transport pathways helped elucidate this further and lets us start to appreciate the full complexity of renal sodium handling. We summarize recent findings in the field in the context of human diseases and a pathophysiologic basis for their treatment.
\end{abstract}

Copyright $@ 2006$ S. Karger AG, Basel

\section{Physiology of Renal Salt Handling}

The preservation of electrolyte homeostasis and thus water balance is vital to our functioning. No idea could be thought, no muscle moved without the proper balance of salts within our body. It is the responsibility of mainly the kidneys to maintain this vital 'milieu interieur'. The primary urine is formed by the glomerular filtrate. Because of their small size, salts fall through the glomerular filter and thus need to be reabsorbed in the renal tubule. The amount to be reabsorbed is quite staggering: assuming a glomerular filtration rate of $100 \mathrm{ml} / \mathrm{min}$ and a serum sodium concentration of $140 \mathrm{mmol} / \mathrm{l}$, an averaged-sized person filters about $20 \mathrm{~mol} / \mathrm{l}$ of sodium per day, equivalent to the amount in $1.2 \mathrm{~kg}$ of cooking salt. Under physiological conditions, renal tubules are capable of reabsorbing $99 \%$ of filtered sodium and water. Without the kidneys sophisticated ability to preserve salt (and water), human life would not exist. This enormous task is accomplished by a combination of distinct and sequentially oriented sodium or sodium-coupled transport systems along the nephron and the concerted and parallel action of some of these systems within the kidney. The active reabsorption of sodium generates the driving force for the passive reabsorption of water.

Genetic or acquired defects in any of these transport systems, which are quite specific and unique for individ-

Robert Kleta, MD, PhD, FASN

SHBG, MGB, NHGRI, NIH, Building 10, Room 10C103C, MSC 1851

10 Center Drive

Bethesda, MD 20892 (USA)

Tel. +1 301451 7979, Fax +1 301402 7290, E-Mail kletar@mail.nih.gov 


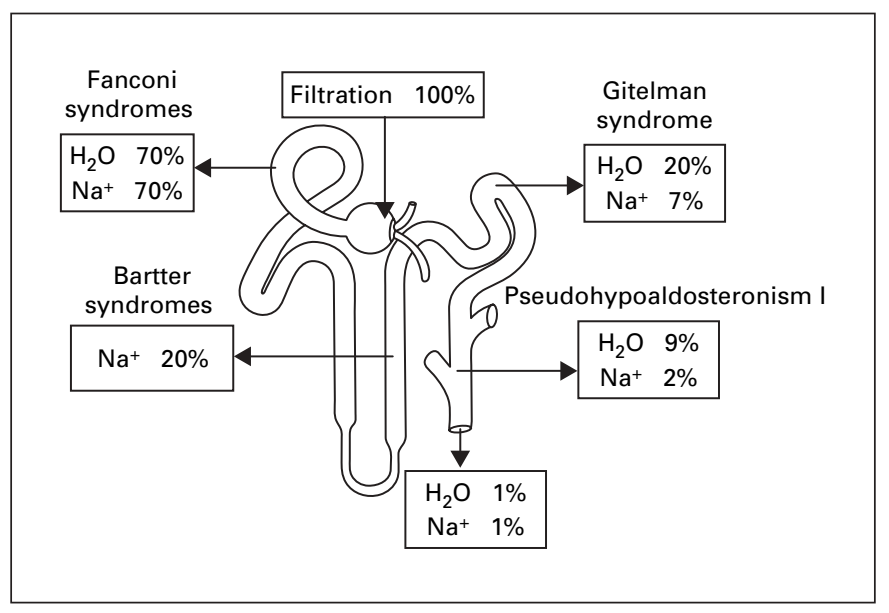

Fig. 1. Sodium and water reabsorption along the nephron. Knockouts of distinctive proteins in particular nephron segments lead to distinctive disease in man as indicated.

ual nephron segments, lead to distinct salt-losing nephropathies (fig. 1). Independently, extrarenal morbidity in salt-losing nephropathies can be caused by either the failure of other ions or compounds of biological importance to be reabsorbed (e.g. phosphate in renal Fanconi syndrome leading to rickets) or the sharing of transporter proteins in different organ systems (e.g. defect in a chloride channel subunit in Bartter syndrome type IV leading to deafness).

Here we will consider the different renal genetic disorders affecting salt (sodium chloride) reabsorption and describe what has recently been learned about them.

\section{Proximal Tubule}

\section{Physiology and Pathophysiology: Renal Fanconi \\ Syndromes}

The proximal tubule (PT) is the part of the nephron where the most diverse action with respect to reabsorption (as well as secretion) takes place (fig. 2). A complete failure has therefore a variety of consequences, amongst which are aminoaciduria, phosphaturia, glucosuria, proximal tubular acidosis, proteinuria and polyuria. Clinical significance is most prominent for phosphaturia, which leads to growth disorders in early childhood and osteomalacia in adults. The bulk of sodium within the kidney and also within the proximal tubule is reabsorbed by means of NHE3, an electroneutral sodium proton exchanger.

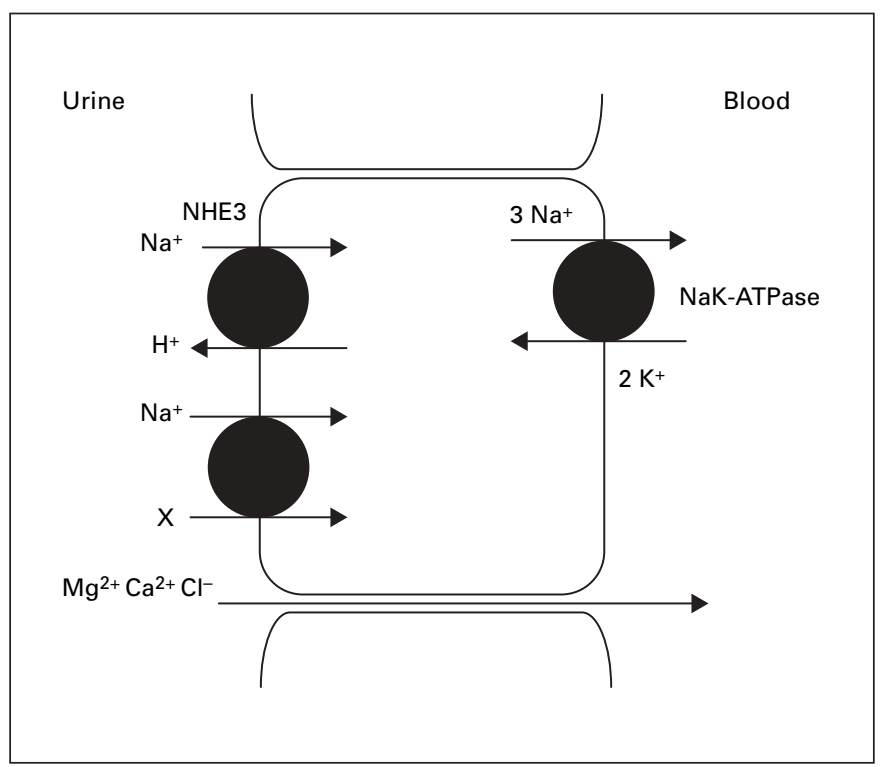

Fig. 2. Simplified diagram of a proximal tubular (PT) cell. Sodium reabsorption in the PT is mainly accomplished by NHE3, which electroneutrally exchanges sodium for protons. Other sodium-coupled transporters use the chemical and electrical gradient of sodium for the reabsorption of molecules (X stands for, e.g., glucose, amino acids, phosphate).

Genetic causes for primary and isolated renal Fanconi syndromes have not been defined yet and, as a result, even nowadays our knowledge about the physiology of the PT is somewhat limited. Interestingly, two different forms of primary autosomal-dominant Fanconi syndrome exist, one with and one without subsequent glomerular kidney failure in adulthood $[1,2]$. Most commonly, a renal Fanconi syndrome occurs secondary to cystinosis, a lysosomal cystine storage disorder. Consequently, in any child presenting with signs or symptoms of Fanconi syndrome, cystinosis has biochemically to be excluded. Partially or full-blown Fanconi syndromes can occur together with several other genetic disorders, e.g. galactosemia, tyrosinemia type I, fructose intolerance, Wilson disease, Fanconi-Bickel syndrome, Lowe syndrome, Dent's disease, ARC syndrome, certain glycogen storage and some mitochondrial disorders. In general, features of the underlying disorder dominate the clinical picture and can make the diagnosis. In contrast, cystinosis initially presents with renal Fanconi syndrome only.

Fanconi syndromes can also occur due to certain intoxications or as side effects of drug treatment, e.g. cadmium, lead, mercury, uranium, glue sniffing, ifosfamide, valproate, gentamicin, fumaric acid, $L$-lysine, suramine, 


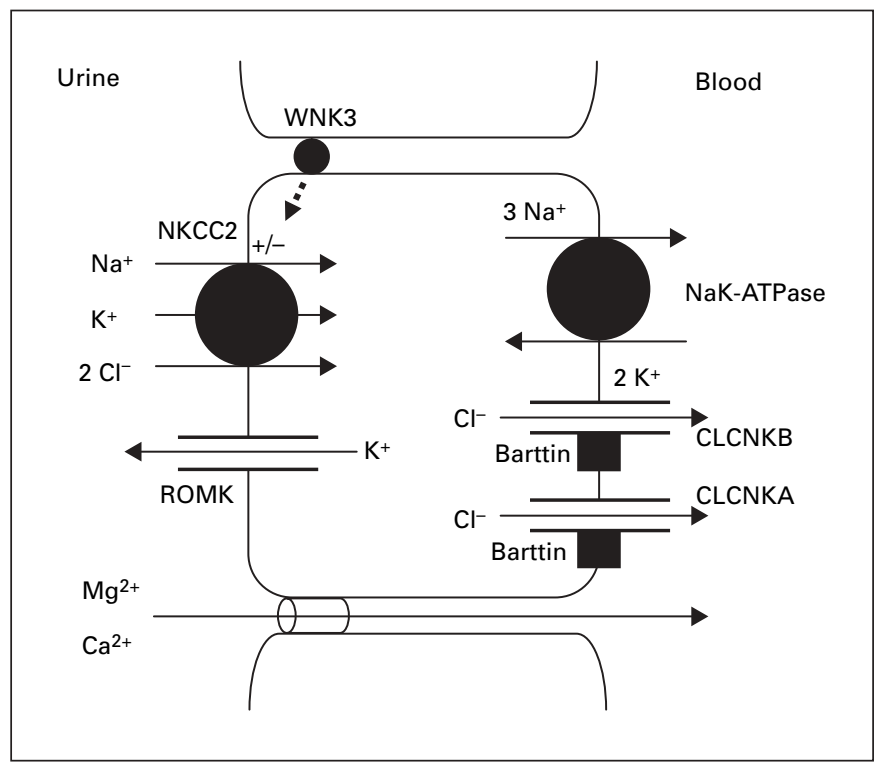

Fig. 3. Simplified diagram of a thick ascending limb (TAL) cell. Sodium is reabsorbed electroneutrally via NKCC2 (defect in Bartter type I), together with one potassium and two chloride ions. The transporter can only function with all 4 ions bound and due to its luminal concentration, potassium binding becomes the rate-limiting step. Therefore, potassium is recycled through the potassium channel ROMK1 (defect in Bartter type II) to ensure an adequate luminal supply of potassium. This also leads to a relative excess of positive charges in the tubular lumen, providing the driving force for paracellular absorption of calcium and magnesium. Sodium can then exit the cell on the basolateral (blood side) via the NaKATPase, while chloride exits through the chloride channels $C L C N K B$ (defect in Bartter type 3) and CLCNKA; both require Barttin (defect in Bartter type 4) for proper membrane localization. WNK3 and a basolateral extracellular calcium sensor regulate NKCC2 activity. NKCC2 can be inhibited by furosemide.

and outdated tetracyclines. It can also been seen in dysproteinemias, e.g. multiple myeloma.

\section{Treatment}

No specific treatment for any of the renal Fanconi syndromes exists. Excessive urinary losses need to be replaced orally to counterbalance fluid, bicarbonate and phosphate deficits. Secondary forms can benefit from specific treatment for the underlying cause, e.g. in cystinosis treatment with cysteamine. Some renal Fanconi syndromes can be abolished by sufficient treatment of the underlying metabolic defect, e.g. galactosemia or tyrosinemia type I. Removing offending drugs can also lead to a reversal of this particular form of tubular nephrotoxicity.

Bartter Syndromes and Other Salt-Losing Tubulopathies

\section{Thick Ascending Limb}

Physiology and Pathophysiology: Bartter Syndromes

The thick ascending limb (TAL) is a major part of the nephron's urine concentration machinery due to its water impermeability and unique sodium chloride reabsorption abilities. Failures here lead inevitably to significant polyuria with all its consequences, especially in infancy and even in utero (polyhydramnios). An electroneutral bumetanide-sensitive (BSC) $\mathrm{Na}-2 \mathrm{Cl}-\mathrm{K}$ cotransporter (NKCC2), encoded by $S L C 12 A 1$, aided by several other proteins is the key element for active transepithelial salt transport in this nephron segment (fig. 3).

Transepithelial transport of sodium chloride in the TAL requires the presence and function of at least 5 gene products. On the luminal membrane, NKCC2 uses the chemical gradient for sodium generated by the basolateral localized sodium potassium ATPase (NaK-ATPase) for the uptake of sodium, chloride and potassium. Furosemide can block this transporter. Potassium has to be recirculated through ROMK into the urine, otherwise it would become the rate-limiting step for reabsorption of sodium chloride. On the basolateral side, sodium exits via the NaK-ATPase, while chloride can exit through at least two chloride channel proteins, predominantly $C L C N K B$, and, to a lesser degree, CLCNKA. Both of these chloride channels require Barttin as subunit to be present for proper function. Mutations in $S L C 12 A 1$, ROMK1, CLCNKB, and $B S D N$ (Barttin) cause autosomal-recessive Bartter syndromes types I, II, III and IV, respectively [3, 4]. Since macula densa cells are derived from the TAL, mutational effects are present here as well. This can lead to an ineffective tubular glomerular feedback (TGF) mechanism, explaining partially the significant disease phenotype in Bartter syndromes. In contrast, Gitelman syndrome is a 'post' macula densa tubular disorder, where the TGF mechanism is undisturbed. Mutations in an extracellular basolateral calcium sensing receptor $(C A S R)$ can cause a dominant Bartter syndrome like picture, but these patients predominant symptoms are hypocalcemic hypercalciuria by which they can be discriminated from Bartter syndrome patients $[3,5]$. Besides $C A S R$, WNK proteins can modulate the activity of luminal transporter proteins in the TAL. WNK3, predominantly expressed at intercellular junctions, was shown to regulate the activity of NKCC2 by altering the expression at the plasma membrane [6].

Patients with Bartter syndromes become clinically apparent already during pregnancy with polyhydramnios (in contrast to diabetes insipidus), or later, in infancy or

Nephron Physiol 2006;104:p73-p80 p75 


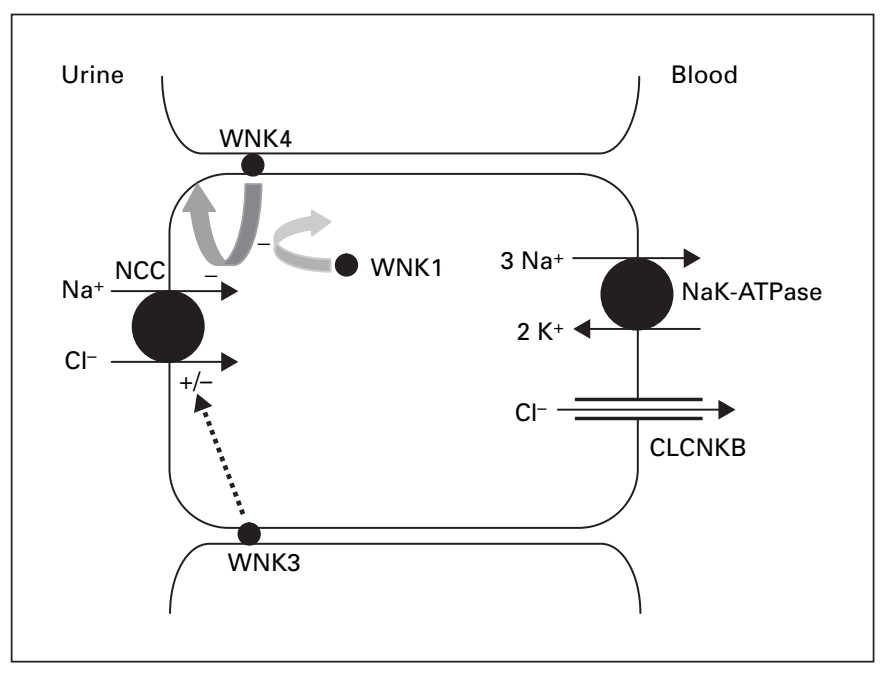

Fig. 4. Simplified diagram of a distal convoluted tubule (DCT) cell. Sodium is reabsorbed electroneutrally via a sodium-chloride cotransporter (NCC) and can then exit towards the blood side via the Na-K-ATPase, while chloride can pass through the basolateral chloride channel $C L C K N B$. WNK1, WNK4, and WNK3 regulate NCC activity. NCC can be inhibited by thiazides.

early childhood with significant polyuria and dehydration. Blood pressures are low normal. Because salt reabsorption in TAL constitutes an important part of the urinary dilution/concentration mechanism, patients with Bartter syndromes type I, II, and IV typically have isosthenuria or even hyposthenuria. The recycling of potassium across the apical membrane of the TAL cell establishes a transepithelial voltage gradient that drives the paracellular absorption of calcium and magnesium (fig. 3). Consequently, Bartter syndromes types I and II are accompanied by hypermagnesiuria and hypercalciuria leading to nephrocalcinosis; types III and IV in general do not show nephrocalcinosis. Chronically, hypokalemia and metabolic alkalosis can be noted due to the compensatory hyperreninemic hyperaldosteronism. Bartter syndrome type II uniquely presents with significant initial neonatal hyperkalemia, which gradually changes to hypokalemia, as ROMK is involved in distal potassium excretion. Later, other potassium channels or transporters can apparently compensate and the patients become hypokalemic. Bartter syndrome type IV presents with sensorineural deafness, which is diagnostic for this type. Usually, Bartter syndrome type III has the mildest course of all Bartter syndrome types, presumably due to the preserved CLCNKA chloride permeability in the TAL. Bartter syndrome type III can also present with signs and symptoms overlapping Bartter and Gitelman syndrome, which is not surprising, considering that this channel is also expressed in DCT (fig. 4). A combined knockout of $C L C N K B$ and $C L C N K A$ leads to a severe form of Bartter syndrome similar to Bartter syndrome type IV [7]. Prostaglandins (e.g. PGE2) are elevated in Bartter syndromes, especially in infancy, and their measurement in the urine can be helpful for diagnosis and treatment. Elevated prostaglandins itself give rise to polyuria, fever, gastrointestinal side effects and need to be addressed pharmacologically (e.g. indomethacin, ibuprofen, COX-2 inhibitors).

\section{Treatment}

Patients with Bartter syndromes face several difficulties for treatment. Significant fluid and sodium chloride losses need to be replaced, which is the more difficult the younger the patient is. Furthermore, chronic and significant hypokalemia poses a challenge for oral potassium replacement therapy. Elevated prostaglandins can induce a vicious cycle for water reabsorption. Since these prostaglandins are principally derived through the action of cyclooxygenase-2 (COX-2), selective and nonselective cyclooxygenase inhibitors can be used for treatment, e.g. celecoxib, ibuprofen, or indomethacin [8]. It is not clear which of these drugs are most beneficial. Often, the use of a certain drug is mandated by the occurrence of side effects of another drug, e.g. gastrointestinal bleeding/ stomach perforation after indomethacin. Not all patients with Bartter syndromes show elevated urinary prostaglandins, especially patients with the mildest form of type III. Other polyuric tubular disorders can also show elevated prostaglandins. However, the measurements of urinary prostaglandins (PGE2) can aid in diagnosis and treatment. Under no circumstances should treatment with thiazides be instituted for hypercalciuria/nephrocalcinosis in these patients since the additional blockade of salt reabsorption in DCT deprives the kidney of an important compensatory mechanism with potentially disastrous consequences. The development of nephrocalcinosis can compromise glomerular function in adult patients and poses a severe problem. Chronic hypokalemia can be addressed pharmacologically with either amiloride or aldosterone antagonists acting in the $\mathrm{CCD}$, but again, these agents impair an important compensatory mechanisms for sodium reabsorption and severe dehydration as a consequence of such a treatment can occur, especially in young children. Patients are encouraged in a high-salt diet, but, in fact, typically have an enormous salt appetite. Moreover, caution is needed for any treatment that promotes rather rapid shifting of extracellular potassium in- 
tracellularly, e.g. inhalative betasympathikomimetika for the treatment of asthma. Some patients with Bartter syndromes show hypomagnesaemia necessitating oral magnesium replacement therapy.

\section{Distal Convoluted Tubule}

\section{Physiology and Pathophysiology: Gitelman}

Syndrome (GS)

The distal convoluted tubule (DCT) has an impact on the reabsorption of several ions, most importantly sodium, calcium and magnesium. Sodium reabsorption in the DCT is mediated by the thiazide-sensitive (TSC) $\mathrm{NaCl}$ cotransporter (NCC), encoded by $S L C 12 A 3$ (fig. 4). Recently, it has been shown that this transporter is regulated through a cascade of two serine threonine kinases, the WNK-kinases WNK1 and WNK4 [9-11]. WNK4 inhibits the transporter, while itself being inhibited by WNK1. Therefore, gain-of-function mutations in WNK1 and loss-of-function mutations in WNK4 lead to excessive NCC-mediated salt-reabsorption with the clinical picture of Pseudohypoaldosteronism type 2 (familial hyperkalaemic hypertension (FHH), Gordon syndrome). These patients suffer from hypertension associated with hyperkalaemia and metabolic acidosis.

Interestingly, while WNK1 is located in the cytoplasm, WNK4 localizes to the tight junctions and has been shown to also regulate paracellular chloride flux and the potassium channel ROMK1 in the collecting duct. It therefore appears that these WNK proteins integrate different epithelial transport pathways to maintain volume and electrolyte homeostasis. Furthermore, WNK3, predominantly expressed at intercellular junctions, was shown to regulate the activity of NCC by altering the expression at the plasma membrane [6].

Impairment of salt re-absorption in the DCT leads to the clinical picture of GS. Patients with GS have decreased salt reabsorption in the DCT caused by mutations in $S L C 12 A 3$ and their clinical symptoms and laboratory findings are best compared with the chronic effects of thiazide diuretics [3]. They typically have low-normal blood pressure and secondary hyperaldosteronism with hypokalemic metabolic alkalosis. In addition, characteristically they have diagnostic hypocalciuria and hypermagnesiuria with subsequent low serum magnesium levels. Interestingly, bone density in these patients increases with age and calcifications in the retina and in cartilage can occur. In contrast to patients with Bartter syndromes, patients with GS typically are diagnosed in late childhood or adulthood with problems such as weakness, tetany or seizures. In other cases, symptoms may be milder and the diagnosis is only made based upon a routine blood test in an asymptomatic patient.

Hypocalciuria and hypermagnesiuria are classical features of GS and their association with inhibition of salt reabsorption in the DCT has long been recognized. In fact, it is used therapeutically in some renal stone patients to minimize urinary calcium excretion. The mechanism underlying this observation however, has been unclear. Recently, some light has been shed on this phenomenon. Through elegant studies using murine knockout models, it was shown that the decrease in calcium excretion is not due to enhanced distal, but rather increased proximal calcium reabsorption where calcium flux parallels sodium reabsorption [12]. Thus, the hypocalciuria is an indirect effect of the NCC-mediated volume depletion. Unfortunately, the molecular pathway for paracellular calcium transport in PT has not been identified yet, and therefore it remains to be shown that inhibition of that pathway would indeed abolish the hypocalciuric effect of NCC inhibition.

The observed hypermagnesiuria is still poorly understood. NCC knockout mice studies and mice treated with thiazides showed decreased expression of the epithelial magnesium channel TRPM6 [12]. This channel mediates the entry step for magnesium from the tubular lumen into the DCT cell. The mechanism, however, for the downregulation of TRPM6 is unknown.

\section{Treatment}

Despite the molecular identification of the defect in GS, no specific therapy is available and thus, treatment is largely symptomatic. Many patients show no signs or symptoms. Patients are encouraged in a high-salt diet, but, in fact, typically have a craving for salt. Supplementations of potassium and magnesium are usually needed. GS patients should not be treated with furosemide since this would represent a drug-induced knockout of the TAL together with a genetic knockout of the DCT.

\section{Cortical Collecting Duct}

\section{Physiology and Pathophysiology: \\ Pseudohypoaldosteronism Type I (PHA-1) and \\ Congenital Adrenal Hyperplasia (CAH)}

At the end of the nephron, the cortical collecting duct (CCD) has the critical role to fine-tune the reabsorption of sodium. Aldosterone in response to renin and angio- 


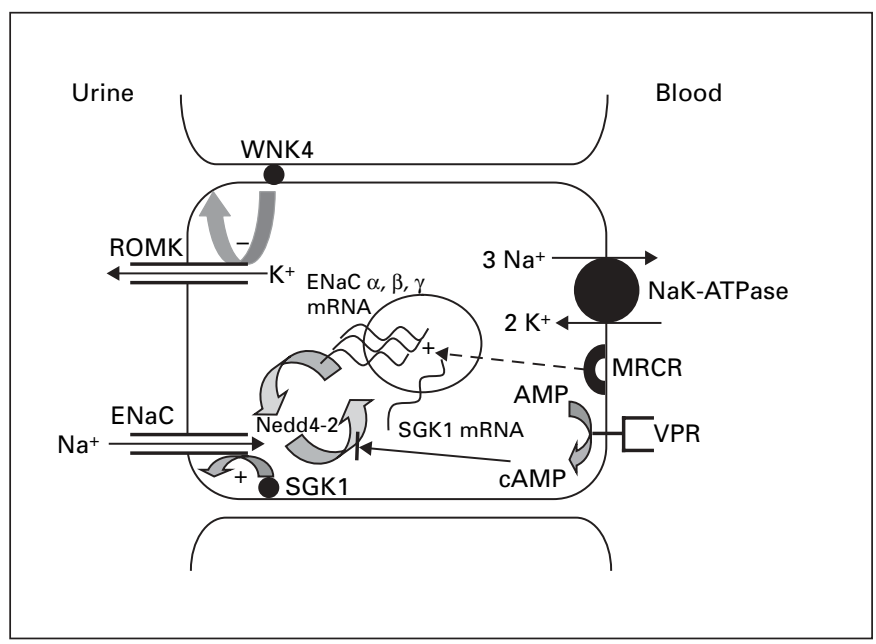

Fig. 5. Simplified diagram of a cortical collecting duct (CCD) principal cell. Sodium reabsorption occurs electrogenic through $\mathrm{ENaC}$ and NaK-ATPase. The activity of these proteins can directly and indirectly be modulated by SGK1, Nedd4-2, MRCR, and VPR. $\mathrm{ENaC}$ can be inhibited by amiloride.

tensin governs the overall expression of critical proteins for this purpose. In addition, the CCD has the critical role of being able to secrete potassium and protons. Furthermore, ADH-regulated water reabsorption aims at maintaining euvolumia. Clinical syndromes with impaired salt reabsorption in the CCD include PHA-1 and CAH. For the purposes of this review, we will only concentrate on the salt wasting aspect of clinical symptoms. Patients with these disorders have an impaired ability to reabsorb salt in the CCD either because of defective mineralocorticoid synthesis, signaling or because of defects in the amiloride sensitive epithelial sodium channel $(\mathrm{ENaC})[3,13,14]$ (fig. 5). Consequently, these patients excrete excessive amounts of sodium in their urine associated with hyperkalaemia and metabolic acidosis. Patients typically manifest during infancy with failure-to-thrive, vomiting and dehydration. In some patients, there is also a history of polyhydramnion due to fetal salt wasting. Patients become particularly ill with intercurrent illnesses, due to their inability to maintain adequate blood pressure.

Mutations in the mineralocorticoid receptor (MRCR) are inherited in an autosomal-dominant fashion and affected patients exhibit typically more mild symptoms. Conversely, mutations in $\mathrm{ENaC}$ subunits are inherited in an autosomal-recessive fashion and patients are more severely affected. Because of the expression of $\mathrm{ENaC}$ in multiple tissues, they also exhibit marked increased salt content in sweat and saliva and have impaired rectal sodium transport. Consequently, their symptoms can overlap with cystic fibrosis.

Sodium is reabsorbed in the collecting duct via $\mathrm{ENaC}$, expressed on principal cells. Expression of $\mathrm{ENaC}$ is under the control of mineralocorticoids, via the MRCR, but also through vasopressin via the vasopressin receptor (VPR) and subsequent production of cyclic AMP (cAMP). Recent evidence suggests an important role for the serum and glucocorticoid-induced kinase (SGK1) in the regulation of ENaC-mediated sodium flux [15]. Aldosterone increases transcription of the genes encoding SGK1, as well as of the $\mathrm{ENaC}$ subunits. Indeed, mice with homozygous deletion of the gene SGK1 have an impaired ability to retain sodium under a sodium-restricted diet [16]. ENaC is mainly regulated through trafficking of the channel. All $\mathrm{ENaC}$ subunits exhibit an amino acid motif in their Cterminal end, called PY-motif, serving as a binding site for a protein called Nedd4-2, which targets ENaC for degradation. In the clinical disorder of Liddle syndrome, this PY motif is disrupted, inhibiting retrieval of $\mathrm{ENaC}$ form the plasma membrane and therefore leading to uncontrolled, excessive sodium reabsorption and hypertension. Nedd4-2 is therefore a convergence point for $\mathrm{ENaC}$ regulation: both aldosterone, as well as vasopressin induces phosphorylation of Nedd4-2, blocking its binding to $\mathrm{ENaC}$.

Reabsorption of sodium in CCD creates the driving force for excretion of potassium and protons, explaining the hyperkalemic metabolic acidosis observed in affected patients.

\section{Treatment}

Salt-losing variants of CAH are due to the adrenal's inability to produce sufficient amounts of mineralocorticoids. Treatment for CAH consists initially mainly of replacement therapy for aldosterone and glucocorticoids.

Treatment of PHA-1 is again symptomatic: patients take salt supplements and typically have a potassium-restricted diet. With intercurrent illnesses, especially vomiting or diarrhea, they often need hospitalization and intravenous replacements of salts and fluid. As with almost all disorders impairing renal sodium reabsorption, symptoms are most severe during the first 1-2 years of life and improve with age. The reasons for this improvement is not clear, but may include a better ability to regulate salt intake (salt appetite) or the maturation or enhanced expression of compensatory mechanisms for tubular salt reabsorption. Certainly, growth favors a less critical water balance. 
Table 1. Genetics of primary renal salt $(\mathrm{NaCl})$ losing nephropathies and their typical clinical characteristics

\begin{tabular}{|c|c|c|c|c|c|c|c|}
\hline Nephron segment & Disorder & OMIM & $\begin{array}{l}\text { Chromo- } \\
\text { some }\end{array}$ & Gene & HUGO alias & $\begin{array}{l}\text { Inheri- } \\
\text { tance }\end{array}$ & $\begin{array}{l}\text { Significant clinical } \\
\text { findings }\end{array}$ \\
\hline \multirow[t]{2}{*}{ Proximal tubule } & $\begin{array}{l}\text { Fanconi syndrome with } \\
\text { kidney failure }\end{array}$ & 134600 & $15 \mathrm{q} 15-\mathrm{q} 22$ & $?$ & & $\mathrm{AD}$ & \multirow{2}{*}{$\begin{array}{l}\text { rickets, glomerular kid- } \\
\text { ney failure in adulthood } \\
\text { rickets, no kidney failure }\end{array}$} \\
\hline & $\begin{array}{l}\text { Fanconi syndrome } \\
\text { without kidney failure }\end{array}$ & 134600 & $?$ & $?$ & & $\mathrm{AD}$ & \\
\hline \multirow{5}{*}{$\begin{array}{l}\text { Thick ascending } \\
\text { limb }\end{array}$} & Bartter syndrome type I & 601678 & $15 \mathrm{q} 21.1$ & SLC12A1 & NKCC2 & $\mathrm{AR}$ & \multirow{5}{*}{$\begin{array}{l}\text { prematurity, nephro- } \\
\text { calcinosis } \\
\text { prematurity, nephro- } \\
\text { calcinosis, postnatal } \\
\text { transient hyperkalaemia } \\
\text { birth at term, no } \\
\text { nephrocalcinosis } \\
\text { prematurity, sensori- } \\
\text { neural deafness, no } \\
\text { nephrocalcinosis } \\
\text { hypocalcemia, } \\
\text { suppressed PTH }\end{array}$} \\
\hline & Bartter syndrome type II & 241200 & $11 \mathrm{q} 24.3$ & KCNJ1 & ROMK1 & AR & \\
\hline & Bartter syndrome type III & 607364 & $1 \mathrm{p} 36.13$ & $C L C N K B$ & $\mathrm{hClC}-\mathrm{Kb}$ & AR & \\
\hline & Bartter syndrome type IV & 602522 & $1 \mathrm{p} 32.3$ & $B S N D$ & BART & $\mathrm{AR}$ & \\
\hline & $\begin{array}{l}\text { hypocalcemia with Bartter } \\
\text { like syndrome }\end{array}$ & 601199 & $3 q 21.1$ & $C A S R$ & $\mathrm{FHH}$ & $\mathrm{AD}$ & \\
\hline $\begin{array}{l}\text { Distal convoluted } \\
\text { tubule }\end{array}$ & Gitelman syndrome & 263800 & $16 \mathrm{q} 13$ & $S L C 12 A 3$ & & $\mathrm{AR}$ & $\begin{array}{l}\text { hypocalciuria, } \\
\text { hypomagnesemia }\end{array}$ \\
\hline \multirow[t]{6}{*}{$\begin{array}{l}\text { Cortical collecting } \\
\text { duct }\end{array}$} & $\begin{array}{l}\text { pseudohypoaldosteronism } \\
\text { type I }\end{array}$ & 177735 & $4 q 31.23$ & $N R 3 C 2$ & MR & $\mathrm{AD}$ & \multirow{6}{*}{$\begin{array}{l}\text { neonatal salt wasting } \\
\text { with hyponatraemia, } \\
\text { hyperkalaemia, acidosis, } \\
\text { remittance with age } \\
\text { neonatal salt wasting } \\
\text { with hyponatremia, } \\
\text { hyperkalaemia, acidosis, } \\
\text { pathological sweat test } \\
\text { neonatal salt wasting, } \\
\text { virilization in females, } \\
\text { pseudopubertas praecox } \\
\text { in males }\end{array}$} \\
\hline & pseudohypoaldosteronism & 264350 & $12 \mathrm{p} 13.31$ & SCNN1A & ENaCalpha & $\mathrm{AR}$ & \\
\hline & type I & & $16 \mathrm{p} 12.2$ & SCNN1B & ENaCbeta & & \\
\hline & & & $16 \mathrm{p} 12.2$ & $S C N N 1 G$ & ENaCgamma & & \\
\hline & congenital adrenal & 201910 & $6 \mathrm{p} 21.32$ & $C Y P 21 A 2$ & $\mathrm{P} 450 \mathrm{c} 21 \mathrm{~B}$ & AR & \\
\hline & hyperplasia & 201810 & $1 \mathrm{p} 12$ & $H S D 3 B 2$ & & & \\
\hline
\end{tabular}

Data from OMIM, HUGO, NCBI (build 35.1). AD = Autosomal dominant; AR = autosomal recessive.

In summary, the study of inherited conditions affecting electrolyte abnormalities and blood pressure have yielded tremendous insight into renal physiology and firmly established the central role of the kidney in the regulation of blood pressure and volume homeostasis. Our knowledge has been further enhanced through the study of genetically manipulated animals. Yet, a lot is still to learn. To give a few examples: (1) The molecular pathway for paracellular calcium flux in PT, for instance, has yet to be defined. (2) Many patients with the clinical picture of Bartter syndromes have no apparent mutation in the four known genes associated with this condition, suggesting that there are more pathways to either mediate or regulate sodium reabsorption in TAL. (3) Another locus for PHA-2 has been described on chromosome 1, suggest- ing another pathway affecting sodium reabsorption in DCT, yet the gene has so far eluded identification. These are only a few selected examples. In fact, the more we learn, the more it becomes obvious, how much is still unknown about the intricate organization and regulation of renal tubular function.

Moreover, the most important promise of molecular medicine, the development of specific treatments targeted precisely at the underlying defect has yet eluded us in the disorders of impaired renal sodium transport. While patients with excessive sodium reabsorption can be treated by specific blockade of their respective pathways, using, for instance, thiazides in PHA-2 or amiloride in Liddle syndrome, there is no specific therapy for the respective loss-of-function disorders. However, with increasing 
knowledge about the precise effect of specific mutations, there may be a silver lining on the horizon. We now understand that a given mutation may not directly affect the function of the encoded molecule, but disturb its proper folding and trafficking. In some disorders of epithelial transport, such as nephrogenic diabetes insipidus, molecular chaperones have been identified, that can induce proper folding in selected mutations and thus rescue trafficking to the membrane [17]. Hopefully, similar progress can be made in the disorders affecting renal sodium transport and ultimately lead to improved treatment of affected patients.

\section{Acknowledgements}

This research was supported in part by the Intramural Research Program of the National Human Genome Research Institute, National Institutes of Health.

\section{References}

1 Lichter-Konecki U, Broman KW, Blau EB, et al: Genetic and physical mapping of the locus for autosomal dominant renal Fanconi syndrome, on chromosome 15q15.3. Am J Hum Genet 2001;68:264-268.

$\checkmark 2$ Tolaymat A, Sakarcan A, Neiberger R: Idiopathic Fanconi syndrome in a family. I. Clinical aspects. J Am Soc Nephrol 1992;2:13101317.

-3 Scheinman SJ, Guay-Woodford LM, Thakker $\mathrm{RV}$, et al: Genetic disorders of renal electrolyte transport. N Engl J Med 1999;340:11771187.

4 Birkenhager R, Otto E, Schurmann MJ, et al: Mutation of BSND causes Bartter syndrome with sensorineural deafness and kidney failure. Nat Genet 2001;29:310-314.

$\checkmark 5$ Watanabe S, Fukumoto S, Chang H, et al: Association between activating mutations of calcium-sensing receptor and Bartter's syndrome. Lancet 2002;360:692-694.
6 Rinehart J, Kahle KT, de Los Heros P, et al: $\mathrm{WNK} 3$ kinase is a positive regulator of $\mathrm{NKCC} 2$ and $\mathrm{NCC}$, renal cation-Cl- cotransporters required for normal blood pressure homeostasis. Proc Natl Acad Sci USA 2005;102:16777_ 16782 .

7 Schlingmann KP, Konrad M, Jeck N, et al: Salt wasting and deafness resulting from mutations in two chloride channels. N Engl J Med 2004; 350:1314-1319.

-8 Kleta R, Basoglu C, Kuwertz-Broking E: New treatment options for Bartter's syndrome. N Engl J Med 2000;343:661-662.

$\checkmark 9$ Kahle KT, Wilson FH, Leng Q, et al: WNK4 regulates the balance between renal $\mathrm{NaCl}$ reabsorption and $\mathrm{K}+$ secretion. Nat Genet 2003;35: 372-376.

10 Kahle KT, Wilson FH, Lifton RP: Regulation of diverse ion transport pathways by WNK4 kinase: a novel molecular switch. Trends Endocrinol Metab 2005;16:98-103.

11 Wilson FH, Disse-Nicodeme S, Choate KA, et al: Human hypertension caused by mutations in WNK kinases. Science 2001;293:11071112 .
12 Nijenhuis T, Vallon V, van der Kemp AW, et al: Enhanced passive $\mathrm{Ca} 2+$ reabsorption and reduced $\mathrm{Mg}^{2+}$ channel abundance explains thiazide-induced hypocalciuria and hypomagnesemia. J Clin Invest 2005;115:1651-1658.

$>13$ Amor M, Parker KL, Globerman H, et al: Mutation in the CYP21B gene (Ile-172-Asn) causes steroid 21-hydroxylase deficiency. Proc Natl Acad Sci USA 1988;85:1600-1604.

$>14$ Rheaume E, Simard J, Morel Y, et al: Congenital adrenal hyperplasia due to point mutations in the type II 3 beta-hydroxysteroid dehydrogenase gene. Nat Genet 1992;1: 239-245.

15 Pearce D, Verrey F, Chen SY, et al: Role of SGK in mineralocorticoid-regulated sodium transport. Kidney Int 2000;57:1283-1289.

16 Wulff P, Vallon V, Huang DY, et al: Impaired renal $\mathrm{Na}(+)$ retention in the sgk1-knockout mouse. J Clin Invest 2002;110:1263-1268.

17 Bernier V, Lagace M, Lonergan M, et al: Functional rescue of the constitutively internalized V2 vasopressin receptor mutant $\mathrm{R} 137 \mathrm{H}$ by the pharmacological chaperone action of SR 49059. Mol Endocrinol 2004; 18:2074-2084. 